Earth Surface Dynamics

Supporting Information for

\title{
Relationships between regional coastal land cover distributions and elevation reveal data uncertainty in a sea-level rise impacts model
}

\author{
Erika E. Lentz, Nathaniel G. Plant, and E. Robert Thieler
}

U.S. Geological Survey Woods Hole and St. Petersburg Coastal and Marine Science Centers

\section{Contents of this file}

Figures $\mathrm{S} 1$

Tables S1-S4

\section{Introduction}

This supplement includes a figure and tables that provide background information on 1) the coastal response model introduced in Lentz et al. (2016), as well as 2) confusion matrices used to compare land cover data sources and predictions of elevation when land cover data were used as inputs (and vice versa). The coastal response model figure is modified from previous publications (Lentz et al., 2015; 2016), and the table shows the general land cover groupings used in Lentz et al. (2016). The confusion matrices show the distributions of data in each category for the full $30 \mathrm{~m} \times 30 \mathrm{~m}$ Northeastern U.S. region, totaling more than 42 million grid cells. The diagonal fields in the confusion matrices (upper left cell to lower right) show where predicted values matched observed values; accuracy rates reported in the captions are calculated as the sum of the diagonal divided by the total number of cells. 


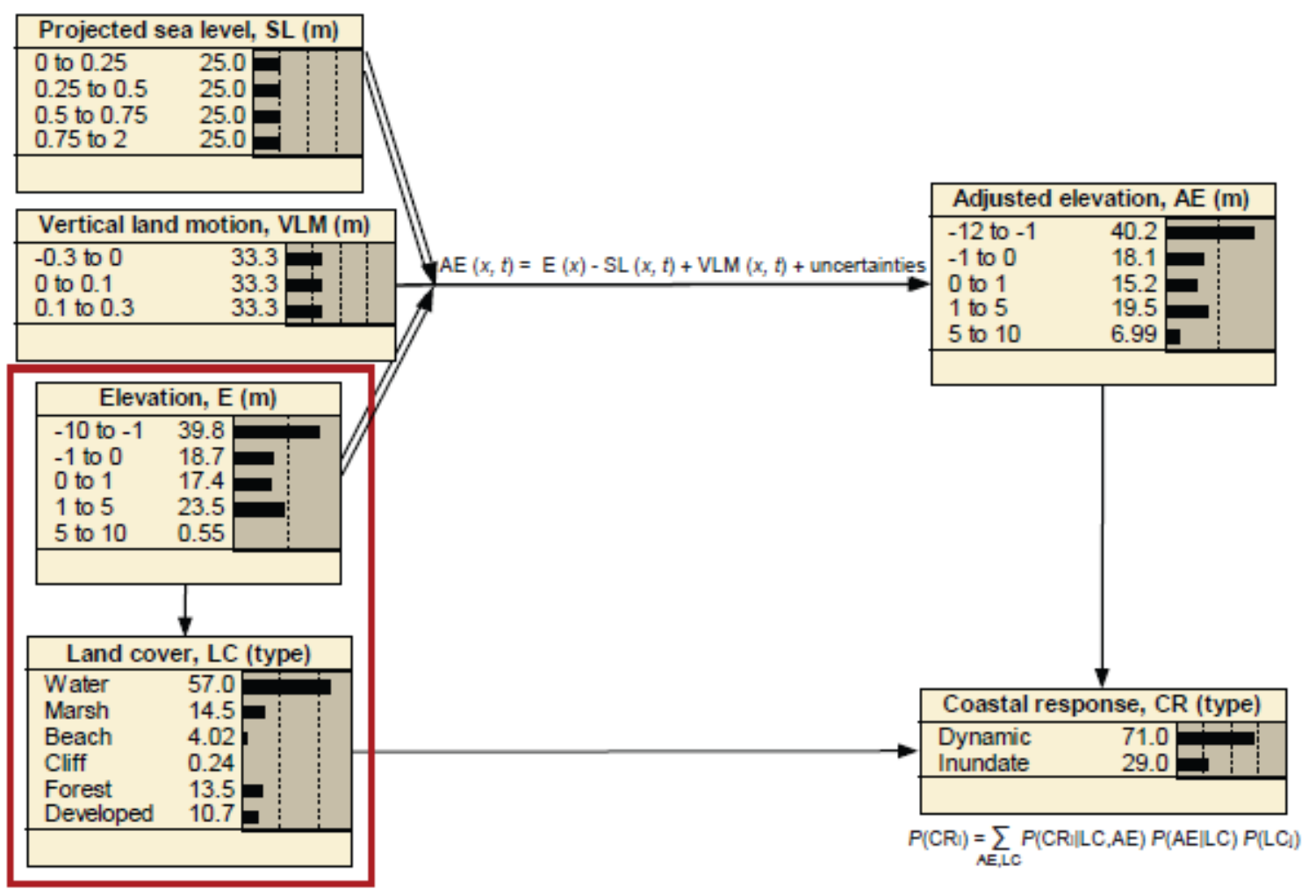

Figure S1. Diagram showing Bayesian network coastal response model, including data inputs (left) and predicted outcomes (right), including adjusted elevation (inundation model equivalent) and coastal response. Horizontal bars shown in the boxes represent prior distributions (probability of occurrence) for each parameter. Uniform distributions for sea-level and vertical land motion parameters provide an equal likelihood of occurrence until a time step is specified. Correlation among nodes are shown by the arrows between them. Equations show deterministic and probabilistic equations used to generate conditional probabilities, where $x$ and $t$ indicate spatial and temporal dependence, and joint correlations of occurrence $i$, at a specific location, j. Red box highlights E-LC relationship trained via Bayes theorem (equation 1) and further tested in this paper. Modified from Lentz et al. $(2015,2016)$. 


\begin{tabular}{|c|c|c|c|}
\hline $\begin{array}{c}\text { Land } \\
\text { Cover } \\
\text { Category* }\end{array}$ & Included DSL Classes* & $\begin{array}{l}\text { CCAP/DSL } \\
\text { comparison }\end{array}$ & Included CCAP Classes \\
\hline Subaqueous & $\begin{array}{l}\text { Bays, lakes, rivers, marine and } \\
\text { estuarine subtidal, and } \\
\text { deepwater }\end{array}$ & Subaqueous & $\begin{array}{l}\text { Open Water, Palustrine Aquatic } \\
\text { Bed, Estuarine Aquatic Bed }\end{array}$ \\
\hline Marsh & $\begin{array}{l}\text { Salt and freshwater marshes, } \\
\text { bogs, swamps, fens, wetland } \\
\text { forests, intertidal aquatic beds, } \\
\text { and reefs }\end{array}$ & Marsh & $\begin{array}{l}\text { Palustrine Forested Wetland, } \\
\text { Palustrine Scrub/Shrub Wetland, } \\
\text { Palustrine Emergent Wetland, } \\
\text { Estuarine Forested Wetland, } \\
\text { Estuarine Scrub/Shrub Wetland, } \\
\text { Estuarine Emergent Wetland }\end{array}$ \\
\hline Beach & $\begin{array}{l}\text { Dune and swale/sandy beach } \\
\text { (including bluffs), marine and } \\
\text { estuarine intertidal } \\
\text { unconsolidated shore }\end{array}$ & \multirow[t]{2}{*}{ Bare Land } & \multirow[t]{2}{*}{ Unconsolidated Shore, Bare Land } \\
\hline Rocky & $\begin{array}{l}\text { Rocky outcrops and shores, } \\
\text { marine and estuarine intertidal } \\
\text { rock bottom }\end{array}$ & & \\
\hline Forest & $\begin{array}{l}\text { Forests, woodlands, } \\
\text { grasslands, agricultural, shrub } \\
\text { lands }\end{array}$ & $\begin{array}{l}\text { Non-Marsh } \\
\text { Vegetation }\end{array}$ & $\begin{array}{l}\text { Cultivated Land, Pasture/Hay, } \\
\text { Grassland, Deciduous Forest, } \\
\text { Evergreen Forest, Mixed Forest, } \\
\text { Scrub/Shrub }\end{array}$ \\
\hline Developed & $\begin{array}{l}\text { All National Land Cover } \\
\text { Dataset developed classes } \\
\text { (open space, low, medium, } \\
\text { and high density), roads, } \\
\text { active and abandoned railroad } \\
\text { tracks }\end{array}$ & Developed & $\begin{array}{l}\text { High Intensity Developed, } \\
\text { Medium Intensity Developed, } \\
\text { Low Intensity Developed, } \\
\text { Developed Open Space }\end{array}$ \\
\hline
\end{tabular}

Table S1. The land-cover classes falling within the six generalized land-cover categories, from Lentz et al. (2015) and as reclassified for use in comparison with Coastal Change Analysis Program (CCAP) data. 


\begin{tabular}{|c|c|c|c|c|c|c|c|}
\hline \multirow[b]{2}{*}{$\begin{array}{l}\text { DSL Land } \\
\text { Cover }\end{array}$} & \multicolumn{6}{|c|}{ C-CAP Land Cover } & \multirow[b]{2}{*}{$\begin{array}{l}\text { User's } \\
\text { accuracy } \\
(\%)\end{array}$} \\
\hline & Subaqueous & Marsh & Bare land & $\begin{array}{l}\text { Non-Marsh } \\
\text { Vegetation }\end{array}$ & Developed & Total & \\
\hline Subaqueous & 22027171 & 625725 & 238956 & 121072 & 170173 & 23183097 & 95 \\
\hline Marsh & 376230 & 5432643 & 40901 & 319470 & 120669 & 6289913 & 86.4 \\
\hline $\begin{array}{l}\text { Bare Land } \\
\text { Non-Marsh }\end{array}$ & 961149 & 386365 & 390012 & 63422 & 39551 & 1840499 & 21.2 \\
\hline Vegetation & 69146 & 1351262 & 47797 & 4509443 & 186375 & 6164023 & 73.2 \\
\hline Developed & 61270 & 454146 & 70835 & 938883 & 3750745 & 5275879 & 71.1 \\
\hline $\begin{array}{l}\text { Ground } \\
\text { truth }\end{array}$ & 23494966 & 8250141 & 788501 & 5952290 & 4267513 & 42753411 & \\
\hline $\begin{array}{l}\text { Producer's } \\
\text { accuracy } \\
(\%)\end{array}$ & 93.8 & 65.8 & 49.5 & 75.8 & 87.9 & & \\
\hline
\end{tabular}

Table S2. Confusion matrix showing comparison between Coastal Change Analysis Program (C-CAP) and Designing Sustainable Landscapes (DSL) land cover datasets with user's error (accuracy) and producer's error (reliability). The overall accuracy rate in this comparison is $85 \%$, where CCAP data are considered as ground truth. 


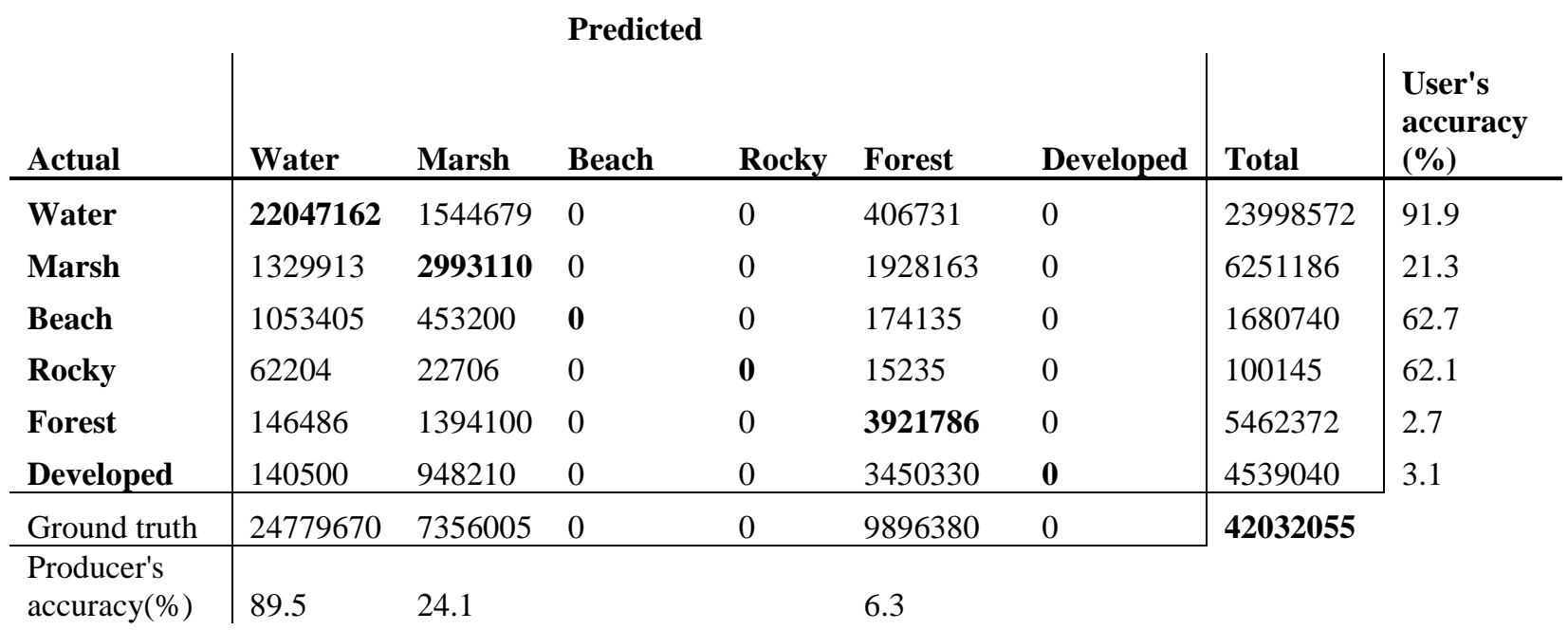

Table S3a. Confusion matrix showing comparison between predicted land cover and measured (observed) land cover when elevation data are used as inputs with original distributions, with user's error (accuracy) and producer's error (reliability). The overall accuracy rate for this comparison is $77 \%$.

\begin{tabular}{l|llllll|l|l}
\multicolumn{7}{c}{} & \multicolumn{7}{c}{ Predicted } \\
Actual & Water & Marsh & Beach & Rocky & Forest & Developed & Total & $\begin{array}{l}\text { User's } \\
\text { accuracy } \\
(\%)\end{array}$ \\
\hline Water & $\mathbf{1 6 5 2 8 3 1 0}$ & 1544679 & 5518852 & 0 & 0 & 406731 & 23998572 & 68.9 \\
Marsh & 61994 & $\mathbf{2 9 9 3 1 1 0}$ & 1267919 & 0 & 0 & 1928163 & 6251186 & 1 \\
Beach & 218448 & 453200 & $\mathbf{8 3 4 9 5 7}$ & 0 & 0 & 174135 & 1680740 & 13 \\
Rocky & 35931 & 22706 & 26273 & $\mathbf{0}$ & 0 & 15235 & 100145 & 35.9 \\
Forest & 11238 & 1394100 & 135248 & 0 & $\mathbf{0}$ & 3921786 & 5462372 & 0.2 \\
Developed & 25627 & 948210 & 114873 & 0 & 0 & $\mathbf{3 4 5 0 3 3 0}$ & 4539040 & 0.6 \\
Ground truth & 16881548 & 7356005 & 7898122 & 0 & 0 & 9896380 & $\mathbf{4 2 0 3 2 0 5 5}$ & \\
\hline $\begin{array}{l}\text { Producer's } \\
\text { accuracy (\%) }\end{array}$ & 98.1 & 24.1 & 70.9 & & & 6.3 & &
\end{tabular}

Table S3b. Confusion matrix showing comparison between predicted land cover and measured (observed) land cover when elevation data are used as inputs with uniform distributions, with user's error (accuracy) and producer's error (reliability). The overall accuracy rate for this comparison is $65.5 \%$ 


\begin{tabular}{|c|c|c|c|c|c|c|c|}
\hline \multirow[b]{2}{*}{ Actual } & \multicolumn{6}{|c|}{ Predicted } & \multirow[b]{2}{*}{$\begin{array}{l}\text { User's } \\
\text { accuracy } \\
(\%)\end{array}$} \\
\hline & -10 to -1 & -1 to 0 & 0 to 1 & 1 to 5 & 5 to 10 & Total & \\
\hline-10 to -1 & 16564241 & 218448 & 61994 & 36865 & 0 & 16881548 & 98.1 \\
\hline-1 to 0 & 5545125 & 834957 & 1267919 & 250121 & 0 & 7898122 & 10.6 \\
\hline 0 to 1 & 1567383 & 453200 & 2966182 & 2342297 & 0 & 7329062 & 40.5 \\
\hline 1 to 5 & 421966 & 174135 & 1928163 & 7372116 & 0 & 9896380 & 74.5 \\
\hline 5 to 10 & 2 & 0 & 26928 & 13 & $\mathbf{0}$ & 26943 & 0 \\
\hline Ground truth & 24098717 & 1680740 & 6251186 & 10001412 & 0 & \multirow{2}{*}{\multicolumn{2}{|c|}{42032055}} \\
\hline $\begin{array}{l}\text { Producer's } \\
\text { accuracy } \\
(\%)\end{array}$ & 68.7 & 49.7 & 47.4 & 73.7 & & & \\
\hline
\end{tabular}

Table S4a. Confusion matrix showing comparison between predicted elevations and measured (observed) elevations when land cover data are used as inputs with original distributions, with user's error (accuracy) and producer's error (reliability). The overall accuracy rate for this comparison is $66 \%$.

\begin{tabular}{|c|c|c|c|c|c|c|c|}
\hline Actual & $\begin{array}{l}\text { Predicted } \\
-10 \text { to }-1\end{array}$ & -1 to 0 & 0 to 1 & 1 to 5 & 5 to 10 & Total & $\begin{array}{l}\text { User's } \\
\text { accuracy } \\
(\%)\end{array}$ \\
\hline-10 to -1 & 16528310 & 254379 & 0 & 36865 & 61994 & 16881548 & 97.9 \\
\hline-1 to 0 & 5518852 & 861230 & 0 & 250121 & 1267919 & 7898122 & 10.9 \\
\hline 0 to 1 & 1544677 & 475906 & $\mathbf{0}$ & 2342297 & 2966182 & 7329062 & 0 \\
\hline 1 to 5 & 406731 & 189370 & 0 & 7372116 & 1928163 & 9896380 & 74.5 \\
\hline 5 to 10 & 2 & 0 & 0 & 13 & 26928 & 26943 & 99.9 \\
\hline Ground truth & 23998572 & 1780885 & 0 & 10001412 & 6251186 & \multirow{2}{*}{\multicolumn{2}{|c|}{42032055}} \\
\hline $\begin{array}{l}\text { Producer's } \\
\text { accuracy (\%) }\end{array}$ & 68.9 & 48.4 & & 73.7 & 30.8 & & \\
\hline
\end{tabular}

Table S4b. Confusion matrix showing comparison between predicted elevations and measured (observed) elevations when land cover data are used as inputs with uniform distributions, with user's error (accuracy) and producer's error (reliability). The overall accuracy rate for this comparison is $59 \%$. 